\title{
SOME SIMPLE DIFFERENTIAL DIFFERENCE EQUATIONS AND THE RELATED FUNCTIONS
}

\author{
H. BATEMAN
}

1. Introduction. The work of Brook Taylor, Jean Bernoulli and Daniel Bernoulli led to the formulation of differential difference equations which are all included in the equation

$$
\begin{aligned}
M d^{2} u_{n} / d t^{2}+ & 2 K d u_{n} / d t+S u_{n} \\
& =(n a+a+b)\left(u_{n+1}-u_{n}\right)-(n a+c)\left(u_{n}-u_{n-1}\right)
\end{aligned}
$$

in which $M, K, S, a, b, c$ are constants. This equation may be treated in at least six different ways, which may be described briefly as follows:

(1) The method of simple solutions in which the aim is to express the desired solution as a sum of simple solutions of type

$$
u_{n}(t)=U_{n}(p) \exp (i p t)
$$

in which $p$ is a constant which may assume a certain set of values. This method was used with great success by the writers just named and was much improved by L. Euler, J. le Rond d'Alembert, J. L. Lagrange and J. Fourier. The method has been greatly developed during the last one hundred fifty years. Some idea of this development may be derived from the excellent report of $\mathrm{H}$. Burkhardt ${ }^{1}$ on expansions in series of oscillating functions.

(2) The method of generating functions in which a differential equation is formed for the generating function

$$
G(z, t)=\sum z^{n} u_{n}(t) .
$$

This method may, perhaps, be associated with the names of Lagrange and Laplace as these writers developed a theory of generating functions. The important developments for the differential difference equation came quite late and began, perhaps, with the work of $\mathrm{Koppe}^{2}$ on the function which I shall call the influence function for

An address presented before the Los Angeles meeting of the Society on November 28,1942 , by invitation of the Program Committee; received by the editors December $26,1942$.

${ }^{1} \mathrm{H}$. Burkhardt, Entwicklungen nach oscillirenden Funktionen und Integration der Differentialgleichungen der mathematischen Physik, Jber. Deutschen Math. Verein. vol. 10 (1908) pp. 1-804.

${ }^{2}$ M. Koppe, Die Ausbreitung einer Erschütterung an der Wellenmaschine durch einen neuen Grenzfall der Besselschen Functionen, Program Andreas-Real-gymnasium, Berlin, No. 96 (1901) 28 pp. See also T. H. Havelock, On the Instantaneous propagation of disturbance in a dispersive medium, Philosophical Magazine (6) vol. 19 (1910) pp. 160-168; E. Schrödinger, Dynamik elastischer gekoppelte Punktsysteme, Annalen 
the equation

$$
d^{2} u_{n} / d t^{2}=u_{n+1}+u_{n-1}-2 u_{n}
$$

(3) The method of definite integrals in which the aim is to express $u_{n}(t)$ in the form of a definite integral such as

$$
u_{n}(t)=\int z^{n} f(z, t) d z
$$

(4) The operational method which depends upon the formation of a difference equation satisfied by the integral

$$
U_{n}(x)=\int_{0}^{\infty} e^{-x t} u_{n}(t) d t
$$

Integrals of this type were used by L. Euler ${ }^{3}$ and P. S. Laplace ${ }^{4}$ to solve differential equations and difference equations by means of definite integrals. The operational method is really the inverse of the transformation of Euler and Laplace since the equation satisfied by $u_{n}(t)$ is known, while in the method of transformation the equation satisfied by $u_{n}(t)$ is derived from an equation satisfied by $U_{n}(x)$. A form of the operational method was used by Poisson ${ }^{5}$ in the solution of two differential difference equations occurring in the theory of the conduction of heat in a bar.

In the case of equation (1.1) the equation for $U_{n}(x)$ is

$$
\begin{aligned}
(n a+a+b)\left(U_{n+1}-U_{n}\right)-(n a+c)\left(U_{n}-U_{n-1}\right) & \\
& +M u_{n}^{\prime}(0)+(2 K-M x) u_{n}(0)=\left(M x^{2}-2 K x+S\right) U_{n} .
\end{aligned}
$$

This is a difference equation. It is homogeneous for $n \neq m$ if the initial conditions are of type

$$
u_{n}(0)=0, \quad n \neq m, \quad u_{m}(0)=1, \quad u_{n}^{\prime}(0)=0 .
$$

For $n=m$ the equation is not homogeneous. The solution of the set

der Physik (4) vol. 44 (1914) pp. 916-934; F. Pollaczek, Über die Fortpflanzung mechanischer Vorgänge in einen linearer Gitter, Annalen der Physik (5) vol. 2 (1929) pp. 991-1011.

${ }^{3}$ L. Euler, De constructione aequationum, Akademiia Nauk, Novi Commentarii vol. 9 (1737) 1744 pp. 85-97; Integralrechnung vol. 2 section II, chaps. 10, 11.

${ }^{4} \mathrm{P}$. S. Laplace, Mémoire sur les approximations des formules qui sont fonctions de très grands nombres, Mémoires de l'Académie Royale des Sciences de Paris (1782; 1785); Oeuvres, vol. 10.

${ }^{5}$ S. D. Poisson, Mémoire sur la distribution de la chaleur dans les corps solides, J. École Polytech. vol. 12 (1823) pp. 1-144. 
of equations may be made unique by adding suitable end conditions. In some cases the condition that $U_{n}(x)$ should be finite for $n= \pm \infty$ is appropriate. Thus for the equation corresponding to (1.3) the $i n$ fluence function is

$$
U_{m, n}(x)=\left(1+x^{2}\right)^{-1 / 2}\left[\left(1+x^{2}\right)^{1 / 2}-x\right]^{2 n-2 m}, \quad n>m .
$$

For $n<m$ the index is $2 m-2 n$. This function $U_{m, n}$ corresponds to Koppe's influence function

$$
u_{m, n}(t)=J_{2(n-m)}(2 t),
$$

and it seems desirable to use the same name in both cases. Recurrence relations which are all of the same type except for one or two values of $n$ are of frequent occurrence in the theory of structures. A case which bears some resemblance to the present one occurs in the work of E. H. Bateman ${ }^{6}$ on the stability of tall building frames. The framework considered by this writer is described as a two-column bent of $N$ stories rigidly connected together, the beams being connected to the columns by joints of variable rigidity. This framework is acted on by a horizontal load at any panel point. The cases of vertical loading on the beams is also considered, the two cases of horizontal and vertical loading being discussed separately.

An influence function for equation (1.1) may be found by the method of the generating function. When the differential difference equation is of the first order the requirement that $u_{n}(0)=0, n=m$, $u_{m}(0)=1$, implies that $G(z, 0)=z^{m}$ and so the arbitrary factor in the solution of the differential equation for $G$ is determined. The influence function for some simple cases is given in the following table:

1. $d u_{n} / d t=u_{n+1}-u_{n}, t^{m-n} e^{-t} / \Gamma(m-n+1)$,

2. $d u_{n} / d t=u_{n-1}-u_{n}, t^{n-m} e^{-t} / \Gamma(n-m+1)$,

3. $2 d u_{n} / d t=u_{n+1}-u_{n-1}, J_{m-n}(t)$,

4. $2 d u_{n} / d t=u_{n+1}+u_{n-1}-2 u_{n}, e^{-t} I_{m-n}(t)$,

5. $2 d u_{n} / d t=u_{n}-2 u_{n+1}+u_{n+2}, e^{t / 2}\left(i t^{1 / 2}\right)^{m-n} H_{m-n}\left(i t^{1 / 2}\right) / \Gamma(m-n+1)$, where in the last case

$$
\exp \left(z t-t^{2} / 2\right)=\sum_{n=0}^{\infty} t^{n} H_{n}(z) / n ! .
$$

$J_{n}(z)$ denotes the Bessel function of order $n, I_{n}(x)$ is the Bessel func-

${ }^{6} \mathrm{E}$. H. Bateman, The stability of tall building frames, Institute of Civil Engineers, London, Selected Paper No. 167, 1934, 49 pp. 
tion of imaginary argument multiplied by the proper factor, $H_{n}(z)$ is the polynomial of Hermite and $\Gamma(z)$ is the Gamma function.

When only the second derivative appears on the left-hand side of the equation the requirement chosen for the definition of the influence function is $u_{n}^{\prime}(0)=0, u_{n}(0)=0, n \neq m, u_{m}(0)=1$, and two additional cases of interest are:

6. $d^{2} u_{n} / d t^{2}=u_{n+1}-u_{n},(t / 2)^{m-n+1 / 2} J_{m-n-1 / 2}(t) / \Gamma(m-n+1)$,

7. $d^{2} u_{n} / d t^{2}=u_{n-1}-u_{n},(t / 2)^{n-m+1 / 2} J_{n-m-1 / 2}(t) / \Gamma(n-m+1)$.

(5) The method of power series. In the case of equation 2 , for in stance, there is a solution of type

$$
\begin{aligned}
u_{n}(t) & =f(n)+\left(t-t_{0}\right)[f(n-1)-f(n)]+\cdots \\
& =\sum_{n=0}^{\infty}\left(t-t_{0}\right)^{m} \Delta^{m} f(n) / m !, \quad \Delta f(n)=f(n-1)-f(n),
\end{aligned}
$$

as noted by G. Doetsch. ${ }^{7}$ The function $f(n)$ is given by the equation $f(n)=u_{n}\left(t_{0}\right)$, and $\Delta^{m} f(n)=u_{n}^{(m)}\left(t_{0}\right)$. Doetsch remarks that when $u_{n}\left(t_{0}\right)$ $=\psi_{0}(n, t)=e^{-t_{0}} t_{0}^{n} / n$ ! we have $u_{n}^{(m)}\left(t_{0}\right)=\left(d^{m} / d t_{0}^{m}\right) \psi_{0}\left(n, t_{0}\right)=\psi_{m}\left(n, t_{0}\right)$ $=p_{m}\left(n, t_{0}\right) \psi_{0}\left(n, t_{0}\right)$, where $p_{m}(x, t)$ is Charlier's polynomial. In this case $u_{n}(t)=\psi_{0}(n, t)$, and $u_{n}^{(m)}(t)=\psi_{m}(n, t)$ is also a solution.

(6) The method of successive approximations. In this method the equation is divided into two parts. One of these is multiplied by a parameter $k$ and a solution is sought which can be expressed as a power series in $k$. The coefficients are determined by a succession of equations which in many cases can be solved very easily. The parameter $k$ is then set equal to 1 after all the operations. In the case of equation 1 , for instance, we use the form $u_{n+1}=k(1+d / d t) u_{n}$ and find that the power series for $u_{n}(t)$ reduces to a single term $u_{n}(t)=k^{n}(1+d / d t)^{n} f(t)$, where $f(t)$ is an arbitrary function which can be differentiated an unlimited number of times. In the case of equation 6 there is a corresponding solution $u_{n}(t)=\left(1+D^{2}\right)^{n} f(t)$ where $D=d / d t$. By choosing $f(t)=t^{-1} \cos t$ we obtain the solution $u_{n}(t)=$ $-(1 / 2) \Gamma(n+1) \Gamma(1 / 2)(t / 2)^{-n-1 / 2} Y_{n+1 / 2}(t)$, and by choosing $f(t)=t^{-1} \sin t$ we get the solution $u_{n}(t)=(1 / 2) \Gamma(n+1) \Gamma(1 / 2)(t / 2)^{-n-1 / 2} J_{n+1 / 2}(t)$, the expressions for the Bessel functions being essentially those of Hargrave and Macdonald. ${ }^{8}$

2. Applications of differential difference equations. The polynomial

${ }^{7}$ G. Doetsch, Die in der Statistik seltener Ereignisse auftretenden Charlierschen Polynome und eine damit zusammenhängende Differentialgleichung, Math. Ann. vol. 109 (1934) pp. 257-266.

${ }^{8}$ See E. T. Whittaker and G. N. Watson, Modern analysis, chap. 17, Ex. 30. 
of Jacques Bernoulli $B_{n}(x)$ satisfies the simple equation

$$
d / d x B_{n}(x)=n B_{n-1}(x)
$$

which is satisfied also by all polynomials of Appell's type. When $B_{n}(x) / n$ ! is denoted by $u_{n}(x)$ the equation is

$$
d u_{n} / d x=u_{n-1} .
$$

This is a particular case of the equation

$$
d u_{n} / d t=k_{n-1} u_{n-1}-k_{n} u_{n}
$$

which occurs in the theory of probability and in the theory of the chain processes of chemistry and radioactivity. The literature on this branch of the subject is now quite large. Equation 2 of $\$ 1$ is also a special case of equation (2.3). Equation 1 may be regarded as the adjoint of equation 2 and it should be noticed that the influence function for this equation is derived from the influence function for equation 2 by interchanging $m$ and $n$. This property is analogous to one which occurs in the theory of the Green's function for a linear differential equation and in the theory of the influence functions of the theory of structures.

In mechanics and in the theory of elasticity the differential difference equations of chief importance are those in which central differences occur. In his interesting book Weather prediction by numerical process, L. F. Richardson emphasizes the advantage of using central differences when difference equations are to be used to furnish approximate solutions of differential equations occurring in the solution of mechanical problems. He was led to this conclusion by some preliminary work on the elastic problem of the masonry dam. An account of this work is given in his paper The approximate arithmetical solution by finite differences of physical problems, Transactions of the Royal Society of London vol. 210A (1910) p. 307.

Following Richardson's lead an attempt will be made here to use the method of divided differences in the solution of various elastic problems. For brevity differential difference equations may be called $D \Delta$-equations. This notation, however, may be thought to denote simply the equations of mixed differences studied by Poisson, Biot and others. A simple case of such an equation

$$
u^{\prime}(x)=a u(x-\theta)-b u(x)
$$

is included in an equation which has become important in economics and has been discussed in several of the recent volumes of the publication Econometrica. 
3. $\mathbf{D} \Delta$-equations in the theory of the compound pendulum. In the case of a pendulum consisting of a light string with equal masses equally spaced and a large mass at the end, the equation of small vibrations is of type

$$
\begin{aligned}
m \ddot{x}_{n}+S x= & (M+n m) g\left(x_{n+1}-x_{n}\right) \\
& -(M+n m-m) g\left(x_{n}-x_{n-1}\right) .
\end{aligned}
$$

For greater generality a spring has been added so that each mass when displaced is acted upon by a restoring force proportional to the displacement $x_{n}$.

In the case of a periodic displacement $x_{n}(t)=X_{n} \cos (p t)$ the difference equation satisfied by $X_{n}$ is of type

$$
(n+s+1) X_{n+1}+(n+s) X_{n-1}=\left(2 n+1+r-k p^{2}\right) X,
$$

where $r$ and $k$ are positive constants.

The equation of motion of the mass $M$ is simply

$$
M \ddot{x}_{0}+S x_{0}=M g\left(x_{1}-x_{0}\right)
$$

and so if $x_{0}=X_{0} \cos (p t)$ the equation connecting $X_{1}$ and $X_{0}$ is

$$
(s+1) X_{1}=\left(1+r-s-k p^{2}\right) X_{0} .
$$

If $k p^{2}=z$ the quantity $X_{n}$ is a polynomial of degree $n$ in $z$.

In the more general case in which the spring attached to the mass $M$ is of different strength $S^{\prime}$, the equation connecting $X_{1}$ and $X_{0}$ is of type

$$
(s+1) X_{1}=\left(q-k p^{2}\right) X_{0},
$$

where $q$ is a constant and $X_{n}$ is again a polynomial of degree $n$ in $z$.

When the string is regarded as of infinite length, the solution of the initial value problem may be solved by finding a weight function $w(z)$ such that

$$
\int_{0}^{\infty} w(z) X_{n}(z) X_{n^{\prime}}(z) d z= \begin{cases}0, & n^{\prime} \neq n, \\ 1, & n^{\prime}=n,\end{cases}
$$

for then if $x_{n}(0)=0, n \neq n^{\prime}, x_{n^{\prime}}(0)=1, x_{n}^{\prime}(0)=0$, we have the formula for the influence function ${ }^{9}$

$$
x_{n^{\prime}, n}(t)=\int_{0}^{\infty} w(z) X_{n}(z) X_{n^{\prime}}(z) \cos (p t) d z=x_{n, n^{\prime}}(t) .
$$

9 The function $x_{n^{\prime}, n}(t)$ has also been called a selective function, see H. Bateman, Selective functions and operations, Amer. Math. Monthly vol. 41 (1934) pp. 556-562. 
The function $w(z)$ of ten may be found by solving the problem of the moments. Since $X_{0}$ is a constant which may be taken to be unity the first equation is

$$
\int_{0}^{\infty} w(z) d z=1 \text {. }
$$

The recurrence relation may be used to find $X_{1}, X_{2}, \cdots$, successively, and as these are polynomials in $z$ the orthogonal relation, with $n^{\prime}=0$, will give the values of the integral

$$
\int_{0}^{\infty} z^{n} w(z) d z, \quad n=1,2,3, \cdots .
$$

In most cases of physical interest the function $w(z)$ is positive for values of $z$ exceeding a certain number $a$ which is either zero or a positive quantity. In the simple case of a pendulum without springs and without the mass $M$ at the end, the function $w(z)$ is $\exp (-z)$ and $X_{n}=L_{n}(z)$ where $L_{n}(z)$ is the polynomial of Daniel Bernoulli, ${ }^{10}$ J. L. Lagrange and E. Laguerre. When the pendulum is suspended from a point at which a mass would ordinarily be concentrated the number $p$ is given by an equation of type $L_{N}(z)=0$, and the initial value problem may be solved by a method indicated by J. L. Lagrange $^{11}$ and Bottema ${ }^{12}$ in which the first essential step is the derivation of an orthogonal relation of type

$$
\sum_{n=0}^{N} L_{n}(z) L_{n}\left(z^{\prime}\right)=0, \quad z \neq z^{\prime},
$$

where $z$ and $z^{\prime}$ are different roots of the equation $L_{N}(z)=0$. The displacement $u_{n}(t)$ is then expressed in the form

$$
u_{n}(t)=\sum_{m=1}^{N}\left[c_{m} L_{n}\left(z_{m}\right) \cos \left(p_{m} t\right)+b_{m} L_{n}\left(z_{m}\right) \sin \left(p_{m} t\right)\right],
$$

the coefficients $b_{m}$ and $c_{m}$ being derived by means of the orthogonal relation. The initial value problem may be solved also by means of the method used for the infinite string if the differential equation is

${ }^{10}$ D. Bernoulli, Akademiia Nauk, Novi Commentarii vol. 6 (1734) p. 108, vol. 7 (1935) p. 162.

${ }^{11} \mathrm{~J}$. L. Lagrange, Méchanique analitique, Part 2, Paris, 1788; Oeuvres, vol. 11, Paris, 1888, section 6. See also Miscellanea Taurinensis, vol. 3 (1762-1765), Oeuvres, vol. 1, p. 534. Lord Rayleigh, Theory of sound, vol. 1 (1877) p. 129.

$12 \mathrm{O}$. Bottema, Die Schwingungen eines zusammengesetzten Pendels, Jber. Deutschen Math. Verein. vol. 42 (1933) pp. 42-60. 
used to continue the values of the initial displacements and velocities to the fictitious masses beyond the point of suspension of the string. This method is generally laborious and may not give simple expressions for the displacement and velocity of the fictitious particles far away from the point of suspension.

It should be remarked that when the solution for initial velocities without initial displacement has been found, the corresponding solution for initial displacements without initial velocities may be derived by differentiation with respect to $t$ in the manner described by the rule of Stokes for the initial value problem for a differential equation of the second order with respect to the time. d'Alembert's solution of the equation $d^{2} u / d t^{2}=d^{2} u / d x^{2}, u=2^{-1} f(x+t)+2^{-1} f(x-t)$ $+(1 / 2) \int_{x-t}^{x+t} g(z) d z$, furnishes a good example for the explanation of the rule. The integral represents the solution of the initial value problem $u=0, d u / d t=g(x)$ and when it is differentiated with respect to $t$ it gives the function $2^{-1} g(x+t)+2^{-1} g(x-t)$, which is the solution of the initial value problem $u=g(x), d u / d t=0$. Replacing $g$ by $f$ in this expression and adding to the integral we have the solution of the general initial value problem $u=f, d u / d t=g$.

4. Derivation of asymptotic forms. The operational method is often useful for the derivation of an asymptotic form of the influence function by means of a method developed by A. Haar, ${ }^{13} \mathrm{G}$. Doetsch ${ }^{14}$ and others. This method is the analogue for integrals of the method of Darboux for finding an estimate for large values of $n$ of the coefficient of $z^{n}$ in the expansion of a function in a power series with a finite radius of convergence. The method of Darboux is based on a study of the behavior of the function around the singularities on the circle of convergence. In the case of an integral of Laplace's type a straight line parallel to the imaginary axis is the boundary of the region of convergence and it is necessary to consider the form of the function represented at the singularities which lie on this line. For example, in the case of the integral

$$
\int_{0}^{\infty} e^{-x t} J_{n}(t) d t=\left(1+x^{2}\right)^{-1 / 2}\left[\left(1+x^{2}\right)^{1 / 2}-x\right]^{n}, \quad R(x) \geqq 0,
$$

there are singularities at $x= \pm i$ on the line $R(x)=0$ and it is readily found that if $s=2 n+1$

${ }^{13}$ A. Haar, Über asymptotische Entwicklungen von Funktionen, Math. Ann. vol. 96 (1926) pp. 69-107.

${ }^{14}$ G. Doetsch, Ein allgemeines Prinzip der asymptotischen Entwicklung, J. Reine Angew. Math. vol. 167 (1932) pp. 274-293. 


$$
\begin{aligned}
J_{n}(t) & \sim(2 \pi t)^{-1 / 2}\left(e^{(i t-i s \pi / 4)}+e^{(i s \pi / 4-i t}\right) \\
& =(2 / \pi t)^{1 / 2} \cos [t-(n+1 / 2) \pi / 2] .
\end{aligned}
$$

Similarly the relation

$$
\int_{0}^{\infty} e^{-x t} d t \int_{0}^{t} J_{n}(z) d z=x^{-1}\left(1+x^{2}\right)^{-1 / 2}\left[\left(1+x^{2}\right)^{1 / 2}-x\right]^{n}, \quad R(x) \geqq 0,
$$

gives the estimate

$$
\int_{0}^{t} J_{n}(z) d z \sim 1+(2 \pi t)^{-1 / 2} \sin [t-(n+1 / 2) \pi / 2] .
$$

These estimates are useful in the discussion of the solution of equation (1.3) which is given by the series

$$
u_{n}(t)=\sum_{m=-\infty}^{\infty} J_{2(n-m)}(2 t) u_{m}(0)+\sum_{m=-\infty}^{\infty} u_{m}^{\prime}(0) \int_{0}^{t} J_{2(n-m)}(2 s) d s .
$$

When $t$ is large each term in the second series gives a finite residual disturbance. These residual disturbances would be eliminated eventually in the case of a finite string by reflections at the ends. The existence of a residual displacement is worth noting, however, as in this respect equation (1.3) differs from its generalization to 2 or more variables of type $n$.

When only one particle has an initial displacement or velocity it is necessary, as Koppe points out, to find an estimate of $J_{2 n}(2 t)$ and its integral for values of $2 n$ and $2 t$, which are of about the same order of magnitude. Some results of this nature were already known but Koppe indicated the advantage of making a substitution of type $x-n=v(n / 6)^{1 / 3}$ when discussing the form of $J_{n}(x)$ for large values of $n$ and $x$.

The investigations relating to the asymptotic form of $J_{n}(x)$ began when Lagrange published his solution of Kepler's problem in the form of a sine series with coefficients of the form $J_{n}(n e)$ and analogous series were obtained by Bessel, Poisson, Hansen and others. Asymptotic forms of the coefficients were needed to decide the question of convergence and notable work was done by Laplace, Poisson, Carlini, Jacobi, Cauchy and Hansen. A second period of investigation began when Debye, Watson and others used the method of steepest descent to estimate the values of definite integrals representing Bessel functions, and methods based on the transformation of differential equations and integrals were employed by Nicholson, Langer, Van Veen, Fock and other writers. Estimates of the integral of the function 
$J_{n}(x)$ have been given by Pollaczek. Some of the results of these investigations are summarized below, the chief terms of the estimates are the only ones given.

$$
\begin{gathered}
x=n \operatorname{sech} a, \quad a>0, \\
J_{n}(x) \sim(2 \pi n \tanh a)^{-1 / 2} e^{-n(a-\tanh a),} \\
\int_{0}^{x} J_{n-1}(w) d w \sim(2 \pi n \tanh a)^{-1 / 2}(\operatorname{coth} a+1) e^{-n(a-\tanh a)}, \\
x=n \sec b, \quad 0 \leqq b \leqq \pi / 4, \\
\left.J_{n}(x) \sim(1 / 2) \pi n \tan b\right)^{-1 / 2} \cos [n(\tan b-b)-\pi / 4], \\
\int_{0}^{x} J_{n-1}(w) d w \sim 1+((1 / 2) \pi n \tan b)^{-1 / 2} \operatorname{cosec} b \\
\cdot \cos [n(\tan b-b)+b-3 \pi / 4], \\
J_{n}(x) \sim(1-b \cot b)\left[\cos (\pi / 6) J_{1 / 3}(n \tan b-n b)\right. \\
\left.-\sin (\pi / 6) Y_{1 / 3}(n \tan b-n b)\right] .
\end{gathered}
$$

If $|x-n| \ll n^{2 / 5}$ Nicholson's estimate is

$J_{n}(x) \sim(6 / x)^{1 / 3} A i\left[(6 / x)^{1 / 3}(x-n)\right], \quad A i(w)=\int_{0}^{\infty} \cos \left(t^{3}-w t\right) d t$

and when $x-n=v(n / 6)^{1 / 3}$ Pollaczek's estimate is

$$
\int_{0}^{x} J_{n-1}(w) d w \sim(1 / 2 \pi i) \int_{C} e^{-t^{3}+v t e i \pi / 3} d t / t .
$$

For these results reference may be made to Burkhardt's report, ${ }^{1}$ Watson's book, ${ }^{15}$ Pollaczek's paper ${ }^{2}$ and the papers of Langer ${ }^{16}$ and Fock. ${ }^{17}$

The following table shows that the maximum of $J_{2 n}(2 t)$ occurs at a time slightly greater than $t=n$.

${ }^{15}$ G. N. Watson, Bessel functions, Cambridge University Press, 1922.

${ }_{16} \mathrm{R}$. E. Langer, On the asymptotic solutions of ordinary differential equations with an application to the Bessel functions of large order, Trans. Amer. Math Soc. vol. 33 (1931) pp. 23-64; On the asymptotic solutions of ordinary differential equations, with an application to the Bessel functions of large complex order, Trans. Amer. Math. Soc. vol. 34 (1932) pp. 447-480.

${ }^{17}$ V. Fock, Neuer asymptotischer Ausdruck für Besselsche Funktionen, C. R. (Doklady) Acad. Sci. U.R.S.S. (2) vol. 1 (1934) pp. 97-102; see also A. Svetlov, Ueber die asymptotischen Ausdrücke fïr Besselsche Funktionen bei grossen Indexen, ibid. (2) vol. 2 (1934) p. 448. 


\begin{tabular}{l|r|r|r|r|r|r|r}
\hline$t$ & 0 & 1 & \multicolumn{1}{c|}{2} & \multicolumn{1}{c|}{3} & \multicolumn{1}{c|}{4} & 5 & 6 \\
\hline$n$ & & & & & & & \\
0 & 1 & .2239 & -.3971 & & & & \\
1 & 0 & .3528 & .3641 & -.2429 & -.1130 & & \\
2 & & .0340 & .2811 & .3576 & -.1054 & & \\
3 & & & .0491 & .2458 & .3376 & -.0145 & \\
4 & & & & .0565 & .2235 & .3179 & \\
5 & & & & & .0608 & .2075 & .3005 \\
\hline
\end{tabular}

5. Surges in springs and connected systems of springs. The use of concentrated masses has been found advantageous in the study of surges in springs such as those of spiral or helical type. Reference may be made to the bibliography in an admirable paper on the subject by K. J. De Juhasz. ${ }^{18}$

An important equation which is a simple generalization of (1.3) is obtained when the concentrated masses on a light string are mounted on springs arranged either along a straight line or on the circumference of a circle or helix. The simplest form of this equation is

$$
d^{2} u_{n} / d t^{2}=u_{n+1}+u_{n-1}-2(1+k) u_{n},
$$

where $k$ is a positive constant. If $G(z, t)=\sum_{n=-\infty}^{\infty} z^{n} u_{n}(t)$ is the generating function $d^{2} G / d t^{2}=\left(z^{-1}+z-2 k-2\right) G$ and if $u_{n}(0)=0, n \neq 0, u_{0}(0)=1$, $u_{n}^{\prime}(0)=0$, an appropriate solution is

$$
G(z, t)=\operatorname{ch}\left[t\left(z^{-1}+z-2 k-2\right)^{1 / 2}\right] .
$$

It is readily seen that $\int_{0}^{\infty} \exp (-x t) G(z, t) d t=x\left(x^{2}+2 k+2-z-z^{-1}\right)^{-1}$. For sufficiently large values of $R(x)$

$$
\int_{0}^{\infty} e^{-x t} u_{n}(t) d t=x\left[\left(x^{2}+2 k+2\right)^{2}-4\right]^{-1 / 2}\left[k+1+x^{2} / 2-y\right]^{n}
$$

where $y^{2}=\left(k+1+x^{2} / 2\right)^{2}-1$.

An application of Haar's method indicates that for large positive values of $t$

$$
\begin{aligned}
& u_{n}(t) \sim(2 \pi t)^{-1 / 2} {\left[(2 k)^{1 / 4} \cos \left\{t(2 k)^{1 / 2}+\pi / 4\right\}\right.} \\
&\left.+(2 k+4)^{1 / 4} \cos \left\{t(2 k+4)^{1 / 2}-\pi / 4-n \pi\right\}\right] .
\end{aligned}
$$

It should be noticed that we also have the equation

${ }^{18} \mathrm{~K} . \mathrm{J}$. De Juhasz, Graphical analysis of surges in mechanical springs, Journal of the Franklin Institute vol. 226 (1938) pp. 505-526, 631-644. The free vibrations of helical springs are treated by the same author in vol. 227 (1939) pp. 647-672. 


$$
\int_{0}^{\infty} e^{-u t^{2}} u_{n}(t) d t=(1 / 2)(\pi / u) \exp (-(k+1) / 2 u) I_{n}(1 / 2 u)
$$

but this expression for the Laplace integral has an essential singularity at the point $u=0$ and is not so convenient for the deduction of the asymptotic form of $u_{n}(t)$. An extension of Haar's method is needed which will make the deduction easy in such a case. In applying Haar's method to equation (5.3) it is advisable to approach each of the four singularities, $x=i(2 k+4)^{1 / 2}, x=i(2 k)^{1 / 2}, x=-i(2 k)^{1 / 2}, x=-i$ $\cdot(2 k+4)^{1 / 2}$, from the right along a line parallel to the real axis. The limiting forms of the arguments of the factors of $\left[\left(x^{2}+2 k+2\right)^{2}-4\right]^{-1 / 2}$ may then be written down for each line of approach without any error.

It is important to notice that in the present case we find by putting $x=0$ in equation (5.3) that

$$
\int_{0}^{\infty} u_{n}(t) d t=0
$$

Hence there is no residual displacement when all the masses are originally in the equilibrium position and the mass numbered 0 is given an initial velocity. The displacement $v_{n}(t)$ at time $t$ is in fact $v_{n}(t)=\int_{0}^{t} u_{n}(s) d s$ and this tends to zero as $t \rightarrow \infty$. This result is quite different from that in Koppe's case when $\int_{0}^{\infty} J_{2 n}(2 s) d s=1 / 2$.

6. The equations of Born and Kármán. In their work on crystal lattices in vibration Born and Kármán ${ }^{19}$ considered equation (1.3) and also a pair of equations which may be written in the form

$$
\begin{aligned}
d^{2} x_{2 n} / d t^{2} & =a^{2}\left(x_{2 n+1}+x_{2 n-1}-2 x_{2 n}\right), \\
d^{2} x_{2 n+1} / d t^{2} & =b^{2}\left(x_{2 n+2}+x_{2 n}-2 x_{2 n+1}\right) .
\end{aligned}
$$

If the initial conditions are $x_{2 n}(0)=0, n \neq m, x_{2 n+1}(0)=1, x_{2 n+1}(0)=0$, $x_{n}^{\prime}(0)=0$, there are two generating functions,

$$
E(z, t)=\sum_{n=-\infty}^{\infty} x_{2 n}(t) z^{2 n}, \quad O(z, t)=\sum_{n=-\infty}^{\infty} x_{2 n+1}(t) z^{2 n+1},
$$

which satisfy the equations

$$
\begin{aligned}
& d^{2} E / d t^{2}=a^{2}\left(z+z^{-1}\right) O-2 a^{2} E \\
& d^{2} O / d t^{2}=b^{2}\left(z+z^{-1}\right) E-2 b^{2} O .
\end{aligned}
$$

19 M. Born and Th. v. Kármán, Ueber Schwingungen in Raumgittern, Physikalische Zeitschrift vol. 13 (1912) pp. 297-309. 
If $m=0$,

$$
\begin{gathered}
d^{2}=a^{2} b^{2}\left(z+z^{-1}\right)^{2}+\left(a^{2}-b^{2}\right)^{2}, \\
d-\left(a^{2}+b^{2}\right)=c^{2}, \quad d+a^{2}+b^{2}=k^{2}, \\
E(z, t)=\left[\left(d+b^{2}-a^{2}\right) / 2 d\right] c h(c t)+\left[\left(d+a^{2}-b^{2}\right) / 2 d\right] \cos (k t), \\
O(z, t)=\left[b^{2}\left(z+z^{-1}\right) / a d\right][c h(c t)-\cos (k t)] .
\end{gathered}
$$

It is advantageous to write

$$
\begin{aligned}
x_{2 n}(t) & =u_{2 n}(t, a, b)+\left(b^{2}-a^{2}\right) v_{2 n}(t, a, b), \\
x_{2 n+1}(t) & =b^{2} v_{2 n}(t, a, b)+b^{2} v_{2 n+2}(t, a, b),
\end{aligned}
$$

where $u_{2 n}$ and $v_{2 n}$ are symmetric functions of $a$ and $b$. The generating functions of $u_{2 n}(t)$ and $v_{2 n}(t)$ are respectively

$$
\begin{aligned}
& U(z, t)=\sum_{n=-\infty}^{\infty} z^{2 n} u_{2 n}(t, a, b)=(1 / 2) \operatorname{ch}(c t)+(1 / 2) \cos (k t), \\
& V(z, t)=\sum z^{2 n} v_{2 n}(t, a, b)=(1 / 2 d)[c h(c t)-\cos (k t)],
\end{aligned}
$$

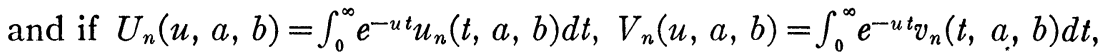

$$
U_{n}(u, a, b)=\left(u^{2}+a^{2}+b^{2}\right) D^{n} E, \quad V_{n}(u, a, b)=D^{n} E,
$$

where $2 a b D=\left(u^{2}+2 a^{2}\right)^{1 / 2}\left(u^{2}+2 b^{2}\right)^{1 / 2}-\left(u^{4}+2 a^{2} u^{2}+2 b^{2} u^{2}\right)^{1 / 2}$, $E=\left[\left(u^{2}+2 a^{2}\right)\left(u^{2}+2 b^{2}\right)\left(u^{2}+2 a^{2}+2 b^{2}\right)\right]^{-1 / 2}$. There is also a relation

$$
\int_{0}^{\infty} e^{-u t} u_{n}(t, a, b) d t / t=D^{n} / 2 n
$$

Use of the multiplication theorem for integrals of Laplace's type gives the addition formula

$$
\int_{0}^{t} v_{m}(t-s, a, b) u_{n}(s, a, b) d s / s=v_{m+n}(t, a, b) / 2 n .
$$

The differential equations satisfied by the functions $u_{2 n}(t, a, b)$, $v_{2 n}(t, a, b)$ are

$$
\begin{gathered}
d^{2} v_{2 n} / d t^{2}=u_{2 n}-\left(a^{2}+b^{2}\right) v_{2 n}, \\
d^{2} u_{2 n} / d t^{2}+\left(a^{2}+b^{2}\right) d^{2} v_{2 n} / d t^{2}=a^{2} b^{2}\left(v_{2 n+2}+v_{2 n-2}-2 v_{2 n}\right) .
\end{gathered}
$$

Elimination of $u$ or $v$ gives the result that $u$ and $v$ both satisfy the differential difference equation

$$
d^{4} u_{2 n} / d t^{4}+2\left(a^{2}+b^{2}\right) d^{2} u_{2 n} / d t^{2}=a^{2} b^{2}\left(u_{2 n+2}+u_{2 n-2}-2 u_{2 n}\right) .
$$

The behavior of $u_{2 n}$ and $v_{2 n}$ for large values of $t$ may be found by 
Haar's method. The result is that if $b>a>0, m=0$,

$$
\begin{gathered}
u_{2 n}(a, b, t)=2^{-3 / 4}(\pi t)^{-1 / 2}\left[( b ^ { 2 } - a ^ { 2 } ) ^ { 1 / 2 } \left\{a^{-1 / 2} b^{-1}\right.\right. \\
\cdot \cos \left[a t 2^{1 / 2}-(2 n+1 / 2) \pi / 2\right]+a^{-1} b^{-1 / 2} \\
\left.\cdot \cos \left(b t 2^{1 / 2}-(2 n-1 / 2) \pi / 2\right)\right\}+\left(\left(a^{2}+b^{2}\right)^{3 / 4} / a b\right) \\
\left.\cdot \cos \left\{t\left(2 a^{2}+2 b^{2}\right)^{1 / 2}-\pi / 4\right\}\right], \\
\cdot \cos \left\{t\left(2 a^{2}+2 b^{2}\right)^{1 / 2}-5 \pi / 4\right\}+\left(1 / a b^{1 / 2}\left(b^{2}-a^{2}\right)^{1 / 2}\right) \\
\cdot \cos \left\{t\left(2 b^{2}\right)^{1 / 2}-3 \pi / 4-n \pi\right\}+\left(1 / b(a)^{1 / 2}\left(b^{2}-a^{2}\right)^{1 / 2}\right) \\
\left.\cdot \cos \left\{t\left(2 a^{2}\right)^{1 / 2}-\pi / 4-n \pi\right\}\right] .
\end{gathered}
$$

Asymptotic forms for $x_{2 n}(t)$ and $x_{2 n+1}(t)$ were worked out by one of my students, C. C. Lin, for the case $b>a$, with the aid of expressions for $\int_{0}^{\infty} e^{-u t} x_{2 n}(t) d t, \int_{0}^{\infty} e^{-u t} x_{2 n+1}(t) d t$. The results agree with those derived from the foregoing formulae. The case $a>b>0$ seemed more difficult but the difficulties are avoided in the present method, as use is made of the symmetry of $u_{2 n}$ and $v_{2 n}$ in $a$ and $b$. Lin's result is that if $b^{2}>a^{2}, c=\left[2\left(a^{2}+b^{2}\right)\right]^{1 / 2}$,

$$
\begin{aligned}
x_{2 n}(t) \sim & (2 / \pi t)^{1 / 2}\left[\left(a / b(c)^{1 / 2}\right) \cos (c t-\pi / 4)\right. \\
& \left.+\left(\left(b^{2}-a^{2}\right)^{1 / 2} / b\left(a(2)^{1 / 2}\right)^{1 / 2}\right) \cos \left(a(t)^{1 / 2}-\pi / 4-n \pi+m \pi\right)\right], \\
x_{2 n+1}(t)= & (2 b / a)(2 / \pi c t)^{1 / 2} \cos (c t-\pi / 4) .
\end{aligned}
$$

Asymptotic forms are needed also for the case in which $c t$ and $2 n$ are both large and of the same order of magnitude. Differential equations for $u_{2 n}(t)$ and $v_{2 n}(t)$ may be derived from those satisfied by their Laplace transforms, but the equations are of high degree and the work of deriving the asymptotic forms from the differential equations has not yet been attempted.

7. Derivation of the solution of an initial value problem with the aid of Poisson's formula for a solution of the wave-equation. Poisson's formula ${ }^{20}$ for a function $w(x, y, z, t)$ which satisfies the wave equation $\partial^{2} w / \partial t^{2}=k^{2}\left(\partial^{2} w / \partial x^{2}+\partial^{2} w / \partial y^{2}+\partial^{2} w / \partial z^{2}\right)$ is

(7.1) $4 \pi w(x, y, z, t)=d / d t\left[t \int_{0}^{\pi} \int_{0}^{2 \pi} d S f(k t)\right]+t \int_{0}^{\pi} \int_{0}^{2 \pi} d S g(k t)$,

${ }^{20} \mathrm{~S}$. D. Poisson, Mémoire pour la propagation du mouvement dans les milieux élastiques, Mémoires de l'Académie des sciences vol. 10 (1823). 
where $f(k t)$ is an abbreviation for the result of replacing $a, b, c$ by $k t \sin \theta \cos \phi, k t \sin \theta \sin \phi, k t \cos \theta$ respectively in the function

$$
f(x+a, y+b, z+c)
$$

and $g(k t)$ has a similar meaning. The symbol $d S$ stands for the elementary solid angle $\sin \theta d \theta d \phi$.

It should be noticed that the function (7.2) is a solution of the 3 partial differential equations

$$
\partial^{2} f / \partial x^{2}=\partial^{2} f / \partial a^{2}, \quad \partial^{2} f / \partial y^{2}=\partial^{2} f / \partial b^{2}, \quad \partial^{2} f / \partial z^{2}=\partial^{2} f / \partial c^{2} .
$$

We now add subscripts $p, q, r$ and assume the function $f$ to be a solution of the additional equations

$$
\begin{aligned}
\partial^{2} f_{p} / \partial a^{2} & =f_{p+1}+f_{p-1}-2 f_{p}, \\
\partial^{2} f_{q} / \partial b^{2} & =f_{q+1}+f_{q-1}-2 f_{q}, \\
\partial^{2} f_{r} / \partial c^{2} & =f_{r+1}+f_{r-1}-2 f_{r} .
\end{aligned}
$$

The function $w(x, y, z, t)$ then becomes $w_{p, q, r}(x, y, z, t)$ and is a solution of the differential difference equation

$$
\begin{array}{r}
d^{2} w_{p, q, r} / d t^{2}=k^{2}\left(w_{p+1, q, r}+w_{p-1, q, r}+w_{p, q+1, r}+w_{p, q-1, r}\right. \\
\left.+w_{p, q, r+1}+w_{p, q, r-1}-6 w_{p, q, r}\right) .
\end{array}
$$

The proof is quite simple because, on account of the equations (7.4), (7.3) satisfied by the function $f_{p, q, r}(x+a, y+b, z+c)$, the righthand side of (7.5) becomes, by virtue of (7.1), the sum of two integrals the first of which has in the integrand

$$
k^{2}\left(\partial^{2} f / \partial x^{2}+\partial^{2} f / \partial y^{2}+\partial^{2} f / \partial z^{2}\right) \quad \text { (subscripts } p, q, r \text { omitted), }
$$

while the second contains a similar expression derived from the function $g_{p, q, r}(x+a, y+b, z+c)$, which is supposed to satisfy the same two sets of equations as $f_{p, q, r}$. When, however, $a, b$, and $c$ have the assigned expressions involving $t, \theta$, and $\phi$ it is a simple consequence of the properties of Poisson's integral that the resulting expression is equal to $\partial^{2} w_{p, q, r} / \partial t^{2}$.

In the particular case in which

$$
\begin{aligned}
& f_{p, q, r}(x+a, y+b, z+c) \\
& \quad=J_{2(p-l)}(2 x+2 a) J_{2(q-m)}(2 y+2 b) J_{2(r-n)}(2 z+2 c)
\end{aligned}
$$

and $g_{p, q, r}(x+a, y+b, z+c)$ has a similar form we obtain a solution which furnishes a solution of the initial value problem for the equation (7.5). Putting $x=0, y=0, z=0$ we write 


$$
\begin{array}{r}
4 \pi I_{p, q, r ; l, m, n}(t)=\frac{d}{d t}\left[t \int_{0}^{\pi} \int_{0}^{2 \pi} \sin \theta d \theta d \phi J_{2(p-l)}(2 k t \sin \theta \cos \phi)\right. \\
\left.\cdot J_{2(q-m)}(2 k t \sin \theta \sin \phi) J_{2(r-n)}(2 k t \cos \theta)\right] .
\end{array}
$$

In the two-dimensional case in which $w$ is independent of $r$ the equation for $w_{p, q}$ is

$$
d^{2} w_{p, q} / d t^{2}=k^{2}\left(w_{p+1, q}+w_{p-1, q}+w_{p, q+1}+w_{p, q-1}-4 w_{p, q}\right),
$$

and the influence function is given by

$$
\begin{array}{r}
4 \pi I_{p, q ; l, m}(t)=\frac{d}{d t}\left[t \int_{0}^{\pi} \int_{0}^{2 \pi} \sin \theta d \theta d \phi J_{2(p-l)}(2 k t \sin \theta \cos \phi)\right. \\
\left.\cdot J_{2(q-m)}(2 k t \sin \theta \sin \phi)\right] .
\end{array}
$$

A change of axes indicates that there is a second formula

$4 \pi I_{p, q ; l, m}(t)=d / d t\left[t \int_{0}^{\pi} \int_{0}^{2 \pi} \sin \theta d \theta d \phi J_{2 p-2 l}(2 k t \sin \theta \cos \phi)\right.$

$$
\left.\cdot J_{2 q-2 m}(2 k t \cos \theta)\right] \text {. }
$$

The integration with respect to $\phi$ can be effected and we get

$$
\text { (7.9) } I_{p, q ; l, m}(t)=\frac{1}{2} \frac{d}{d t}\left[t \int_{0}^{\pi} \sin \theta d \theta J_{p-l}^{2}(k t \sin \theta) J_{2 q-2 m}(2 k t \cos \theta)\right] \text {. }
$$

This is the simplest expression that has been obtained for the function $I$. The method of the generating function indicates that

$$
\text { (7.91) } x^{l} y^{m} \operatorname{ch}\left[k t\left(x+x^{-1}+y+y^{-1}-4\right)^{1 / 2}\right]=\sum_{p=-\infty}^{\infty} \sum_{q=-\infty}^{\infty} I_{p, q ; l, m}(t) x^{p} y^{q} \text {. }
$$

If we write

$$
\left(x+x^{-1}+y+y^{-1}-4\right)=\left(x^{1 / 2}-x^{-1 / 2}\right)^{2}+\left(y^{1 / 2}-y^{-1 / 2}\right)^{2},
$$

a power series for $I$ may be obtained. On the other hand, if we multiply the generating function by $\exp \left(-z t^{2}\right)$ and integrate with respect to $t$ from 0 to $\infty$, the left-hand side of equation (7.91) becomes

$$
(1 / 2)(\pi / z)^{1 / 2} \exp k^{2}\left(x+x^{-1}+y+y^{-1}-4\right) / 4 u,
$$

and so 


$$
2 \int_{0}^{\infty} e^{-z t^{2}} I_{p, q ; l, m}(t) d t=(\pi / z)^{1 / 2} e^{-k^{2} / z} I_{p-l}\left(k^{2} / 2 z\right) I_{q-m}\left(k^{2} / 2 z\right) .
$$

When $u$ is large and positive $I_{n}(u) \sim(2 \pi u)^{-1 / 2} e^{u}$ and so when $z \rightarrow 0$ we obtain the equation

$$
\int_{0}^{\infty} I_{p, q ; l, m}(t) d t=0
$$

Now the solution of the initial value problem is

$$
w_{p, q}(t)=\sum I_{p, q ; l, m}(t) w_{l, m}(0)+\sum \int_{0}^{t} d s I_{p, q ; l, m}(s) d s w_{l, m}^{\prime}(0),
$$

consequently there is no residue (as $t \rightarrow \infty$ ) arising from an initial velocity imparted to one of the particles, and the associated displacement of each particle ultimately approaches zero.

It may be noted that the function $I_{p, q}(t) \equiv I_{p, q ; 0,0}(t)$ satisfies the partial difference equation

$$
q\left(I_{p+1, q}-I_{p-1, q}\right)=p\left(I_{p, q+1}-I_{p, q-1}\right),
$$

which is the analogue of the equation $y \partial F / \partial x=x \partial F / \partial y$ which expresses that $F$ depends only on $x^{2}+y^{2}$.

When it is desired to extend the present analysis to the equation $\partial^{2} w / \partial t^{2}+\partial^{4} w / \partial x^{4}+2 \partial^{4} w / \partial x^{2} \partial y^{2}+\partial^{4} w / \partial y^{4}=0$, which occurs in the theory of a vibrating plate, use may be made of the approximations to partial derivatives of high order given in the paper of E. Pflanz. ${ }^{21}$ The expression which replaces the derivatives of the fourth order then consists of 25 terms. References are given to the work of other writers on this subject.

8. Elastic waves in a lattice. Taking first the case of motion in two dimensions we replace the usual equations of motion by differential difference equations. Let $u_{p, q}, v_{p, q}$ be the components of displacement at the lattice point $(p, q)$ and let $P, Q$ be operators such that

$$
P u_{p, q}=u_{p+1 / 2, q}-u_{p-1 / 2, q}, \quad Q u_{p, q}=u_{p, q+1 / 2}-u_{p, q-1 / 2} ;
$$

then if $\lambda, \mu$ are the elastic constants of Lamé the components of stress may be defined to be

$$
\begin{aligned}
& \overline{x x}_{p, q}=2 \mu P u_{p, q}+\lambda\left(P u_{p, q}+Q v_{p, q}\right), \\
& \overline{y y}_{p, q}=2 \mu Q v_{p, q}+\lambda\left(P u_{p, q}+Q v_{p, q}\right), \\
& \overline{x y}_{p, q}=\overline{y x}_{p, q}=\mu\left(P v_{p, q}+Q u_{p, q}\right),
\end{aligned}
$$

${ }^{21}$ E. Pflanz, Über die Annäherung linearer partieller Differentialausdriicke durch finite Ausdr ïcke, Jber. Deutschen Math. Verein. vol. 48 (1938) pp. 41-48. 
and the equations of motion are

$$
\begin{aligned}
\rho d^{2} u_{p, q} / d t^{2} & =\mu P^{2} u_{p, q}+(\lambda+\mu)\left(P^{2} u_{p, q}+P Q v_{p, q}\right)+\mu Q^{2} u_{p, q} \\
\rho d^{2} v_{p, q} / d t^{2} & =\mu P^{2} v_{p, q}+(\lambda+\mu)\left(P Q u_{p, q}+Q^{2} v_{p, q}\right)+\mu Q^{2}{ }_{p, q} .
\end{aligned}
$$

These may be satisfied by writing

$$
\begin{aligned}
& u_{p, q}=A \alpha a^{p} b^{q} e^{i w t}+B \gamma a^{p} c^{q} e^{i w t}, \\
& v_{p, q}=A \beta a^{p} b^{q} e^{i w t}-B \alpha a^{p} c^{q} e^{i w t},
\end{aligned}
$$

where $A, B, a, b, c, w$ are arbitrary constants and $\alpha=a^{1 / 2}-a^{-1 / 2}$, $\beta=b^{1 / 2}-b^{-1 / 2}, \gamma=c^{1 / 2}-c^{-1 / 2}$. Substitution in the equations of motion indicates, however, that these will be satisfied when $a, b, c, w$ are related to $\rho, \lambda, \mu$ in such a way that

$$
-\rho w^{2}=(\lambda+2 \mu)\left(\alpha^{2}+\beta^{2}\right), \quad-\rho w^{2}=\mu\left(\alpha^{2}+\gamma^{2}\right) .
$$

The boundary condition will be taken to be $\left(\overline{y y}_{p, q}\right)=0, \overline{x y}_{p, q}=0$, for $q=0$. This gives the two conditions $A\left[2 \mu \beta^{2}+\lambda\left(\alpha^{2}+\beta^{2}\right)\right]=2 \mu B \alpha \gamma$, $2 A \alpha \beta=B\left(\alpha^{2}-\gamma^{2}\right)$. Elimination of $A$ and $B$ gives the equation

$$
\left[2 \mu \beta^{2}+\lambda\left(\alpha^{2}+\beta^{2}\right)\right]\left(\alpha^{2}-\gamma^{2}\right)=4 \mu \alpha^{2} \beta \gamma \text {. }
$$

When the values of $\beta$ and $\gamma$ are inserted a relevant solution of this equation is

$$
w=-i V_{R} \alpha,
$$

where $V_{R}$ is the velocity of the Rayleigh wave. If $a=e^{i z}, b=e^{-r}$, $c=e^{-s}$ we have

$\beta=2 \operatorname{sh}(r / 2), \quad \gamma=2 \operatorname{sh}(s / 2), \quad \alpha=2 i \sin (z / 2), \quad w= \pm 2 V_{R} \sin (z / 2)$.

Also if $V_{L}$ is the velocity of propagation of longitudinal waves, $V_{T}$ the velocity of propagation of transverse waves,

$$
\begin{aligned}
& w^{2} / V_{L}^{2}=4 \sin ^{2}(z / 2)-4 \operatorname{sh}^{2}(r / 2), \\
& w^{2} / V_{T}^{2}=4 \sin ^{2}(z / 2)-4 \operatorname{sh}^{2}(s / 2) .
\end{aligned}
$$

The expressions for the displacements now take the form $\beta=-2 \operatorname{sh}(r / 2), \gamma=-2 \operatorname{sh}(s / 2), \alpha=2 i \sin (z / 2), w= \pm 2 V_{R} \sin (z / 2)$

$$
\begin{aligned}
u_{p, q}= & C(z)\left(2-V_{R}^{2} / V_{T}^{2}\right) e^{i\left(p z \pm 2 V_{R} t \sin (z / 2)\right)-q r} \\
& -(1 / 2) C(z)\left(2-V_{R}^{2} / V_{L}^{2}\right)^{2} e^{i\left(p z \pm 2 V_{R} t \sin (z / 2)\right)-q s}, \\
v_{p, q}= & +i C(z)\left(2-V_{R}^{2} / V_{T}^{2}\right)\left(1-V_{R}^{2} / V_{L}^{2}\right)^{1 / 2} e^{i\left(p z \pm 2 V_{R} t \sin (z / 2)\right)-q r} \\
& +2 i C(z)\left(1-V_{R}^{2} / V_{L}^{2}\right)^{1 / 2} e^{i\left(p z \pm 2 V_{R} t \operatorname{siu}(z / 2)\right)-q s}
\end{aligned}
$$


where $C(z)$ is an arbitrary function of $z$. Taking this to be unity and integrating with respect to $z$ between 0 and $2 \pi$ we obtain a type of influence displacement, the values of $u_{p, 0}$ and $v_{p, 0}$ being

$$
\begin{aligned}
& u_{p, 0}=(1 / 2)\left(V_{R}^{2} / V_{T}^{2}\right)\left(2-V_{R}^{2} / V_{T}^{2}\right)\left[\bar{J}_{2 p}\left(2 V_{R} t\right)+i \bar{E}_{2 p}\left(2 V_{R} t\right)\right] \\
& v_{p, 0}=\left(V_{R}^{2} / V_{T}^{2}\right)\left(1-V_{R}^{2} / V_{L}^{2}\right)^{1 / 2}\left[\bar{E}_{2 p}\left(2 V_{R} t\right)-i \bar{J}_{2 p}\left(2 V_{R} t\right)\right]
\end{aligned}
$$

where

$$
\begin{aligned}
& \bar{J}_{2 p}(x)=\frac{1}{2 \pi} \int_{0}^{2 \pi} \cos (p \theta \pm x \sin \theta / 2) d \theta=J_{2 p}(\mp x), \\
& \bar{E}_{2 p}(x)=\frac{1}{2 \pi} \int_{0}^{2 \pi} \sin (p \theta \pm x \sin \theta / 2) d \theta=E_{2 p}(\mp x) .
\end{aligned}
$$

$J_{v}(x)$ is the Bessel function of order $v$ when $v$ is an integer and $E_{v}(x)$ is Weber's function. When $x$ is large and positive the estimates given in Watson's Bessel functions ${ }^{15}$ are

$$
\begin{aligned}
& J_{v}(x) \sim(\pi x / 2)^{-1 / 2} \cos \left(x-\frac{2 v+1}{4} \pi\right), \\
& E_{v}(x) \sim(\pi x / 2)^{-1 / 2} \sin \left(x-\frac{2 v+1}{4} \pi\right) .
\end{aligned}
$$

It should be noticed that $\bar{E}_{2 p}(0)=0, \bar{J}_{2 p}(0)=0, p \neq 0, \bar{J}_{0}(0)=1$. An initial displacement of the lattice point $(0,0)$ leaves no residue at any surface point as $t \rightarrow \infty$.

A method of finding an estimate of $E_{v}(x)$ when both $x$ and $v$ are large is indicated in Watson's Bessel functions.

California Institute of Technology 